\title{
Interactions of commonly used dietary supplements with cardiovascular drugs: a systematic review
}

Salmaan Kanji ${ }^{1}$, Dugald Seely ${ }^{1,2}$, Fatemeh Yazdi ${ }^{3}$, Jennifer Tetzlaff ${ }^{3}$, Kavita Singh ${ }^{3}$, Alexander Tsertsvadze ${ }^{3}$, Andrea C Tricco ${ }^{4}$, Margaret E Sears ${ }^{3,5}$, Teik C Ooi ${ }^{6}$, Michele A Turek ${ }^{7}$, Becky Skidmore ${ }^{7}$ and Mohammed T Ansari ${ }^{3^{*}}$

\begin{abstract}
Background: The objective of this systematic review was to examine the benefits, harms and pharmacokinetic interactions arising from the co-administration of commonly used dietary supplements with cardiovascular drugs. Many patients on cardiovascular drugs take dietary supplements for presumed benefits and may be at risk for adverse supplement-drug interactions.

Methods: The Allied and Complementary Medicine Database, the Cochrane Library, EMBASE, International Bibliographic Information on Dietary Supplements and MEDLINE were searched from the inception of the review to October 2011. Grey literature was also reviewed.

Two reviewers independently screened records to identify studies comparing a supplement plus cardiovascular drug(s) with the drug(s) alone. Reviewers extracted data using standardized forms, assessed the study risk of bias, graded the strength of evidence and reported applicability.

Results: Evidence was obtained from 65 randomized clinical trials, 2 controlled clinical trials and 1 observational study. With only a few small studies available per supplement, evidence was insufficient for all predefined gradable clinical efficacy and harms outcomes, such as mortality and serious adverse events. One long-term pragmatic trial showed no benefit from co-administering vitamin E with aspirin on a composite cardiovascular outcome. Evidence for most intermediate outcomes was insufficient or of low strength, suggesting no effect. Incremental benefits were noted for triglyceridemia with omega-3 fatty acid added to statins; and there was an improvement in levels of high-density lipoprotein cholesterol with garlic supplementation when people also consumed nitrates
\end{abstract}

Conclusions: Evidence of low-strength indicates benefits of omega-3 fatty acids (plus statin, or calcium channel blockers and antiplatelets) and garlic (plus nitrates or warfarin) on triglycerides and HDL-C, respectively. Safety concerns, however, persist.

Keywords: Cardiovascular drugs, Dietary supplements, Harms, Systematic review

\section{Background}

The American Heart Association estimates that more than 81 million (one in three) American adults have at least one form of cardiovascular disease (CVD) [1]. Pharmaceutical interventions are the front line therapies for the prevention and treatment of CVD in addition to lifestyle and dietary recommendations [2-4].

\footnotetext{
* Correspondence: moansari@ohri.ca

${ }^{3}$ Ottawa Methods Centre, Clinical Epidemiology Program, Ottawa Hospital Research Institute, University of Ottawa Evidence-based Practice Center, Ottawa, ON, Canada

Full list of author information is available at the end of the article
}

Billions of dollars are spent annually in the US on complementary and alternative medicine, and a large portion of this expenditure is directly on dietary supplements [5]. Approximately one-third to nearly two-thirds of people experiencing CVD use some form of complementary and alternative medicine that includes dietary supplements, and are thus at risk for potential adverse events from interactions with pharmacologically active agents, and non adherence associated with polypharmacy [6-11]. Evidence of both benefit and harms of

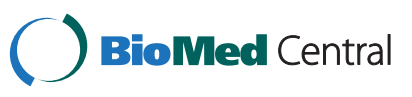


adding a supplement to cardiovascular (CV) drugs has been reported [12,13].

While much research is available describing drug-drug interactions in various populations, the evidence is less well described for drug-supplement interactions or simply the effects of add-on supplementation, especially in populations with CVD. The aim of this extensive synthesis review is to examine the evidence for benefits, harms, pharmacokinetic and statistical interactions from coadministration of a set of commonly used dietary supplements with CV drugs.

\section{Methods}

We followed a pre-specified and peer-reviewed study protocol. The Agency for Healthcare Research and Quality (AHRQ) commissioned the full evidence report, which is available online [14].

\section{Data sources and searches}

Using a peer-reviewed strategy, the Allied and Complementary Medicine Database, the Cochrane Library, EMBASE, International Bibliographic Information on Dietary Supplements and MEDLINE, as well as the grey literature, were searched from study inception until October 2011.

\section{Study selection}

A comparative study was eligible if it was published in English or German; other languages were excluded due to concerns with study quality [15] or applicability [16]. We included German language publications given the well-developed regulations for research, practice and use of dietary supplements in Germany [17-19]. We included studies where a dietary supplement was coadministered with a CV drug(s) compared with the drug alone or co-administered with another supplement, and clinical or surrogate CV efficacy or harms, or pharmacokinetic outcomes in any adult population were reported. A dietary supplement was defined as a vitamin, mineral, herb or botanical, amino acid, concentrate or metabolite or extract, enzymes or tissues intended for ingestion in a pill, capsule, tablet, powder or liquid form not represented for use as a conventional food or as the sole item of a meal or diet [20]. We selected to review specific supplements of interest based on reported surveys and input from public and independent technical expert panels [6,21-26]. We aimed to restrict to common supplements and CV medications taken by adults and elderly for which current evidence on possible drugsupplement interaction was lacking. Studies were also included which evaluated the use of coenzyme Q10, Echinacea, garlic, ginger, Ginkgo biloba, Panax ginseng, American ginseng ( $P$. quinquefolius), hawthorn, oral magnesium, niacin ( $\leq 250 \mathrm{mg} /$ day $)$, omega-3 fatty acids or fish oils, red yeast rice extract, resveratrol, vitamin A, vitamin $\mathrm{D}$ with or without calcium, vitamin $\mathrm{E}$ or vitamin $\mathrm{K}$ as supplements. Finally, we included studies that employed CV drugs commonly used in outpatient settings in the US and Canada. Additional file 1: Table S1.

One reviewer screened all titles and abstracts for potential relevance, and a second verified exclusions at this level. Two independent reviewers assessed the full publication of any potentially relevant studies, with discrepancies resolved by consensus.

\section{Data extraction and quality assessment}

Study characteristics, population, intervention, comparator and outcomes data were extracted using standardized forms. The extracted outcomes of interest were categorized into four groups: clinical, intermediate, harms and pharmacokinetic (for example, area under the curve (AUC), half life of $\mathrm{CV}$ drug, maximum of peak concentration (Cmax), and amount of time that a drug is present at the maximum concentration (tmax)). The full list of outcomes considered for this review is presented in Additional file 2: Table S2. One reviewer with a clinical background rated study populations with respect to 10-year coronary heart disease (CHD) risk (high, moderate and low) according to the National Cholesterol Education Program Adult Treatment Panel III guidelines [27]. When all participants were healthy non-smokers, study level 10-year CHD risk was categorized as low. We assessed risks of bias according to outcome, using generic items for confounding, selection, performance, detection and attrition bias. Certain quality criteria were specific to particular study designs (for example, allocation generation and concealment applied only to randomized clinical trials (RCTs)). The overall study risk of bias for pre-specified gradable outcomes Additional file 1: Table S1 and Additional file 2: Table S2 was rated as low, moderate or high, and then independently verified. Similarly, the strength of the body of evidence [28] and the applicability of the evidence for outcomes with conclusive results [29] were rated according to the published guidance.

\section{Grading the strength of evidence}

A methodologist and a content expert graded the strength of the body of evidence per each outcome (high, moderate, low or insufficient) based on the following four domains: overall risk of bias, consistency, directness and precision [28]. Only a set of pre-specified important outcomes identified a priori through consultations with the Technical Expert Panel was graded Additional file 3: Table S3. This was done because customarily only a subset of important outcomes is chosen to grade the strength of evidence - outcomes that are 
more meaningful for decision-making given a specific research question [28].

The strength of evidence was graded as insufficient when there was no evidence for an outcome, the direction of the estimates was inconsistent across studies, and/or the evidence from the contributing study/studies had an imprecise statistically non-significant pooled estimate (the 95\% confidence intervals (CIs) were wide enough to be compatible with either clinical benefit, true no difference or harm).

\section{Data synthesis and analysis}

All analyses compared the combination of a dietary supplement plus CV drug(s) with CV drug(s) alone or plus placebo or plus another dietary supplement.

The decision to pool individual study results was based on the degree of similarity in methodological and clinical characteristics of studies under consideration. Metaanalysis was considered when studies were randomized trials that included similar populations, compared the same type of dietary supplement and CV medication in the intervention and control groups, and reported the identical outcome measures in the same statistical format (for example, mean difference (MD) or geometric mean ratio (GMR)). The estimates of post-treatment $\mathrm{MD}$ for continuous outcomes and relative risk (RR) for binary outcomes (except for rare events) of individual studies were pooled using a random-effects model by DerSimonian and Laird [30]. The choice of this model was based on the assumption that some residual clinical and methodological diversity might still exist across the pooled studies despite the similarities [31]. For dichotomous outcomes with event rates $<1 \%$, the Peto odds ratios (ORs) based on a fixed effects model were calculated. The results from studies with zero events in one of the arms were pooled using the fixed effects MantelHaenszel method without continuity correction. Studies with zero events in both arms were not pooled [31].

The degree of statistical heterogeneity across pooled studies was assessed by visual inspection of the forest plot and the generation of Cochran's $Q(\alpha=0.10)$ and the $\mathrm{I}^{2}$ statistic. We planned to explore clinical (for example, gender, age, ethnicity) and methodological factors (for example, parallel versus crossover design, risk of bias, type of analysis, baseline health status) as potential sources of statistically significant heterogeneity (Chisquare statistic $P<0.10$ and/or $\mathrm{I}^{2}>50 \%$ ).

We followed the US Food and Drug Administration (FDA) guidance for analysis and interpretation of drug interaction pharmacokinetic studies; the two comparators are considered bioequivalent (no clinically important difference) if the lower and upper bounds of the 90\% CI for the GMR were between 0.8 and 1.25 [32]. Where applicable, we examined statistical interactions between supplements and drugs by calculating the synergy index [33].

All analyses were performed using Comprehensive Meta Analysis version 2.2.057, StatsDirect statistical software and R: A Language and Environment for Statistical Computing, Foundation for Statistical Computing [34-36].

\section{Role of the funding source}

The AHRQ supported this study but had no role in formulating study questions, conducting the systematic review, or approving the manuscript for submission and publication.

\section{Results}

In total, 32,314 records were screened for eligibility (Figure 1). Sixty-three unique English language studies, including one of observational design, contributed evidence to the present review regarding the interactions of supplement-drug combinations [37-99]. We found no relevant unique German publications, nor a good quality systematic review to obviate the need for de novo synthesis of evidence. Most studies had a moderate risk of bias for the gradable outcomes. A paucity of studies precluded exploration of subgroup heterogeneity and how this may have affected outcomes. Statistical interactions between supplement(s) and drug(s) were generally not reported.

Here, we outline selected results for gradable outcomes Additional file 3: Table S3 from the systematic review [100] limited to American ginseng, coenzyme Q10, Echinacea, garlic, Ginkgo biloba, omega-3 fatty acids and vitamin $\mathrm{E}$. While the full report commissioned by AHRQ can be found at http://www.effectivehealthcare.ahrq.gov/ index.cfm) [101], selected results reported here are limited to those that provide some signal of evident or potential drug interaction.

Overall, evidence for clinical, harms and pharmacokinetic outcomes was inconclusive (grade: insufficient). The majority of the reported evidence was based on intermediate efficacy outcomes (grade: insufficient to low).

These results represent evidence on: clinical outcomes of effectiveness from 19 RCTs [37-55] (Table 1); intermediate surrogate outcomes from 52 RCTs and two nonRCTs [38,39,41,43,45,48-94] (Tables 2 and 3); harms identified from $52 \mathrm{RCTs}$ and a single retrospective cohort study [37-41,43,45-48,50-68,70-73,76-78,80-84,87,90-99] (data available online[100]); and lastly direct evidence of pharmacokinetic data on interactions between supplements and CV drugs from 11 RCTs $[37,60,63,66,71,83,96,98]$ (data available online [100]).

Overall, the majority of included RCTs were of moderate risk of bias with only $25 \%$ of the studies explicitly reporting adequate sequence generation and $9 \%$ reporting allocation concealment. Adherence to $\mathrm{CV}$ medication was 


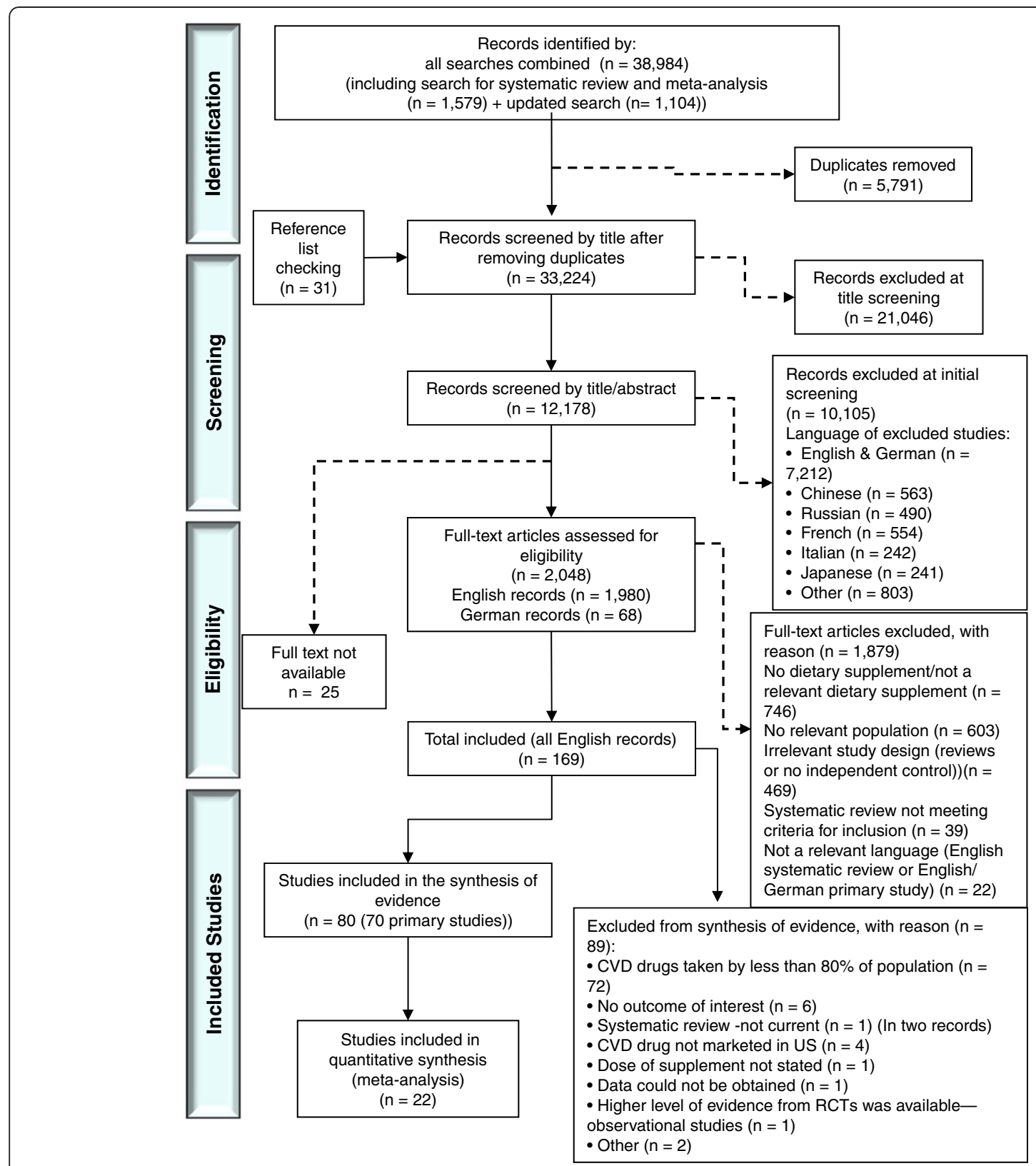

Figure 1 Preferred Reporting Items for Systematic Reviews and Meta-Analyses (PRISMA). Summary of evidence search and selection

reported for co-administration of coenzyme Q10 plus statins, omega-3 fatty acids plus statins, and vitamin E plus statins in five studies [38-40,43,52].

\section{American ginseng (panax quinquefolius) Clinical outcome efficacy}

No evidence.

\section{Intermediate outcome efficacy or harms}

Evidence from three RCTs for co-administration of ginseng and warfarin was inconclusive (grade: insufficient) [68-70].

\section{Pharmacokinetic outcomes}

American ginseng ( 2 g/day from weeks 2 to 4 ) caused a statistically significant reduction in the warfarin AUC (between group difference in median change from week 1 to week $4,-0.64 \mu \mathrm{g} / \mathrm{mL}$ per day (95\% CI $-1.25,-0.13)$ ) [69]. The clinical significance of this finding is unclear because the analysis was not based on GMRs.

\section{Coenzyme Q10}

\section{Clinical outcome efficacy}

Evidence for the effect of coenzyme Q10 co-administered with angiotensin-converting enzyme inhibitors on all- 
Table 1 Gradable clinical outcomes for dietary supplements plus cardiovascular drugs ${ }^{\mathrm{a}}$

\begin{tabular}{|c|c|c|c|c|}
\hline Item & $\begin{array}{l}\text { Supplement } \\
\text { (dose) }\end{array}$ & Cardiovascular drug & $\begin{array}{l}\text { Number of studies, sample size, } \\
\text { characteristic (treatment duration) }\end{array}$ & $\begin{array}{l}\text { Outcome } \\
\text { (combination versus control) }\end{array}$ \\
\hline \multicolumn{5}{|l|}{$\begin{array}{l}\text { Clinical outcome } \\
\text { efficacy }\end{array}$} \\
\hline \multirow[t]{6}{*}{ All-cause mortality } & $\begin{array}{l}\text { Coenzyme Q10 } \\
(100 \mathrm{mg} / \mathrm{day})\end{array}$ & $\begin{array}{l}\text { ACE inhibitors ( } 80 \% \text { of } \\
\text { participants were also } \\
\text { taking digoxin, furosemide, } \\
\text { hydralazine and/or nitrates) }\end{array}$ & $\begin{array}{l}\text { Single study [53]; } 30 \text { mostly male patients } \\
\text { with left ventricular dysfunction ( } 3 \text { months) }\end{array}$ & Death: none versus one \\
\hline & $\begin{array}{l}\text { Ginkgo biloba } \\
\text { ( } 40 \mathrm{mg} \text { four } \\
\text { times a day) }\end{array}$ & Antiplatelet agents & $\begin{array}{l}\text { Single study [47]; } 62 \text { South Asian patients with } \\
\text { previous ischemic stroke ( } 1 \text { month) }\end{array}$ & No deaths \\
\hline & $\begin{array}{l}\text { Omega-3 fatty } \\
\text { acids ( } 4 \mathrm{~g} / \text { day) }\end{array}$ & Aspirin & $\begin{array}{l}\text { Single study [46]; } 291 \text { patients admitted for } \\
\text { coronary artery bypass grafting (1 year) }\end{array}$ & Death: five versus four \\
\hline & & Statins & $\begin{array}{l}\text { Single study [37]; } 50 \text { healthy nonsmoking } \\
\text { adults ( } 2 \text { weeks) }\end{array}$ & No deaths \\
\hline & & Warfarin & $\begin{array}{l}\text { Single study [46]; } 319 \text { patients admitted } \\
\text { for coronary artery bypass grafting ( } 1 \text { year) }\end{array}$ & Deaths: three versus two \\
\hline & & Fenofibrate & $\begin{array}{l}\text { Single study [55]; } 167 \text { participants with } \\
\text { hyperglycemia ( } 2 \text { months) }\end{array}$ & No deaths \\
\hline Quality of life & $\begin{array}{l}\text { Coenzyme Q10 } \\
\text { (100 mg/day) }\end{array}$ & ACE inhibitors & $\begin{array}{l}\text { Single study [53]; } 30 \text { mostly male patients } \\
\text { with left ventricular dysfunction ( } 3 \text { months) }\end{array}$ & $\begin{array}{l}\text { Minnesota 'Living with } \\
\text { Heart Failure' questionnaire } \\
\text { (mean sum of all scores } \\
\text { post-treatment } 26.7 \pm 17.9 \\
\text { versus } 26.5 \pm 18.7\end{array}$ \\
\hline \multirow[t]{2}{*}{$\begin{array}{l}\text { Myocardial } \\
\text { infarction }\end{array}$} & $\begin{array}{l}\text { Omega-3 fatty } \\
\text { acids }(1.8 \mathrm{~g} \\
\text { eicosapentaenoic } \\
\text { acid }+1.2 \mathrm{~g} \\
\text { docosahexaenoic } \\
\text { acid) }\end{array}$ & $\begin{array}{l}\text { Aspirin + calcium channel } \\
\text { antagonists }\end{array}$ & $\begin{array}{l}\text { Single study [50]; } 58 \text { patients who had } \\
\text { undergone successful coronary angioplasty } \\
\text { ( } 6 \text { months) }\end{array}$ & Acute Ml: 4 versus 2 \\
\hline & & & & RR $1.70(95 \%$ Cl 0.32, 8.84) \\
\hline Arrhythmia & $\begin{array}{l}\text { Omega-3 fatty } \\
\text { acids ( } 4 \text { g/day) }\end{array}$ & Statins & $\begin{array}{l}\text { Single study [41]; } 256 \text { patients with persistent } \\
\text { hypertriglyceridemia despite statin therapy } \\
\text { ( } 2 \text { months) }\end{array}$ & Arrhythmia: one versus none \\
\hline Stroke & $\begin{array}{l}\text { Vitamin } \mathrm{E} \\
(0.4 \mathrm{~g} / \text { day })\end{array}$ & Aspirin & $\begin{array}{l}\text { Single study [48]; } 100 \text { patients with previous } \\
\text { reversible or irreversible ischemic neurologic } \\
\text { deficit ( } 2 \text { years) }\end{array}$ & $\begin{array}{l}\text { Fatal or non-fatal stroke: } \\
\text { three versus six }\end{array}$ \\
\hline $\begin{array}{l}\text { Ischemic stroke, } \\
\text { hemorrhagic stroke } \\
\text { and transient } \\
\text { ischemic attack }\end{array}$ & $\begin{array}{l}\text { Vitamin E } \\
(600 \mathrm{IU} / \text { day })\end{array}$ & Aspirin & $\begin{array}{l}\text { Single study [40]; } 19,934 \text { healthy women } \\
\text { (10 years) }\end{array}$ & $\begin{array}{l}\text { Composite outcome of } \\
\text { nonfatal Ml, nonfatal stroke } \\
\text { and vascular death, RR } 0.95 \\
(95 \% \mathrm{Cl} 0.79,1.13)\end{array}$ \\
\hline
\end{tabular}

Inconclusive results: studies had an imprecise statistically non-significant pooled estimate (the $95 \%$ Cls were wide enough to be compatible with either clinical benefit, true no difference or harm). ACE: angiotensin-converting enzyme; Cl:confidence interval; MI:myocardial infarction; RR: relative risk.

cause mortality and quality of life in one study of mostly male patients with left ventricular dysfunction was inconclusive (grade: insufficient) [53]. No differences in adherence to statins were noted with co-administration of coenzyme Q10 [39].

\section{Intermediate outcome efficacy}

Low grade evidence was available from one trial indicating no significant difference in high-density lipoproteincholesterol (HDL-C) for combination of coenzyme Q10 plus fenofibrate versus fenofibrate alone [56].

\section{Harms}

Evidence from five RCTs for the combination of coenzyme Q10 and statins, fenofibrate or angiotensin- converting enzyme inhibitors was inconclusive (grade: insufficient) $[39,53,56-58]$.

\section{Pharmacokinetic outcomes}

No evidence.

\section{Echinacea}

Clinical outcome efficacy

No evidence.

\section{Intermediate outcome efficacy or harms}

Evidence from one study for the combination of Echinacea and warfarin was inconclusive (grade: low) [97]. 
Table 2 Gradable intermediate outcomes for dietary supplements plus cardiovascular drugs (low grade evidence)

\begin{tabular}{|c|c|c|c|c|}
\hline $\begin{array}{l}\text { Outcome } \\
\text { measures }\end{array}$ & Dietary supplement & $\begin{array}{l}\text { Cardiovascular } \\
\text { drug(s) }\end{array}$ & Conclusion effect estimate & Applicability \\
\hline \multirow[t]{5}{*}{$\begin{array}{l}\text { Lipid } \\
\text { profile }\end{array}$} & Co-Q10 (200 mg/day) & Fenofibrate & $\begin{array}{l}\text { No difference for HDL-C (one study): MD, } 1.55 \mathrm{mg} / \mathrm{dL} \\
(95 \% \mathrm{Cl}-6.78,3.68)\end{array}$ & Mean age: \\
\hline & & & & 53 years \\
\hline & & & & Mixed gender \\
\hline & & & & High CHD risk \\
\hline & & & & 12 weeks treatment \\
\hline \multirow{3}{*}{$\begin{array}{l}\text { Lipid } \\
\text { profile }\end{array}$} & Garlic (4 g/day) & Nitrates & In favor of combination & Unknown age, gender \\
\hline & & & HDL-C (one study): MD, 8.40 mg/dL (95\% Cl 1.91, 14.89) & High CHD risk \\
\hline & & & & 12 weeks treatment \\
\hline \multirow[t]{7}{*}{$\begin{array}{l}\text { Lipid } \\
\text { profile }\end{array}$} & $\begin{array}{l}\text { Omega-3-fish oil } \\
\text { ( } 3.6 \mathrm{~g} / \text { day omega-3 } \\
\text { to } 9.2 \mathrm{~g} / \text { day fish oil) }\end{array}$ & Statins & In favor of combination & Mean age: 45 to 63 years \\
\hline & & & $\begin{array}{l}\text { TG (two studies pooled): MD, }-74.95 \mathrm{mg} / \mathrm{dL} \\
(95 \% \mathrm{Cl}-95.80,-54.10)^{\mathrm{a}}\end{array}$ & Mixed $\mathrm{CHD}$ risk \\
\hline & & & No difference between combination and CV drug alone & Mixed gender \\
\hline & & & HDL-C (six studies pooled): MD, 2.26 mg/dL $(95 \% \mathrm{Cl}-1.8,6.3)$ & Up to 25 weeks treatment \\
\hline & & & LDL-C (five studies pooled): MD, $1.3 \mathrm{mg} / \mathrm{dL}(95 \% \mathrm{Cl}-3.6,6.2)$ & \\
\hline & & & Achieving LDL-C targets: RR $0.93(95 \%$ Cl 0.84, 1.03) & \\
\hline & & & Achieving HDL-C targets (one study): and $1.00(95 \% \mathrm{Cl} 0.90,1.10)$ & \\
\hline \multirow[t]{2}{*}{$\begin{array}{l}\text { Lipid } \\
\text { profile }\end{array}$} & $\begin{array}{l}\text { Omega-3-fish oil } \\
(1.8 \mathrm{~g} / \text { day })+\end{array}$ & $\begin{array}{l}\text { Calcium channel } \\
\text { blockers + aspirin }\end{array}$ & In favor of combination & Mean age: 57 y; \\
\hline & & & $\begin{array}{l}\text { TG (two studies not pooled): MD }-81.00 \mathrm{mg} / \mathrm{dL} \\
(95 \% \mathrm{Cl}-125.30,-36.70) \text { and } \mathrm{MD}-54.00 \mathrm{mg} / \mathrm{dL} \\
(95 \% \mathrm{Cl}-94.1,-13.90)\end{array}$ & $85 \%$ men \\
\hline
\end{tabular}

\begin{tabular}{|c|c|c|c|c|}
\hline & & & & High CHD risk \\
\hline & & & & Up to 6 weeks treatment \\
\hline \multirow[t]{5}{*}{$\begin{array}{l}\text { Lipid } \\
\text { profile }\end{array}$} & $\begin{array}{l}\text { Omega-3-fish oil } \\
\text { (3.2 g/day) }\end{array}$ & $\begin{array}{l}\text { Calcium channel } \\
\text { blockers + aspirin, } \\
\text { or dipyridamole }\end{array}$ & In favor of CV drug alone & Mean age: 56 y; \\
\hline & & & LDL-C (one study): MD 21.00 mg/dL (95\% Cl 3.30, 38.70) & $100 \%$ men \\
\hline & & & In favor of combination & High $\mathrm{CHD}$ risk \\
\hline & & & TG (one study): MD -81.0 mg/dL (95\% Cl -125.30, -36.70) & \\
\hline & & & & Up to 12 weeks treatment \\
\hline \multirow[t]{5}{*}{$\begin{array}{l}\text { Lipid } \\
\text { profile }\end{array}$} & $\begin{array}{l}\text { Vitamin E } \\
\text { (900 mg/day) }\end{array}$ & Nifedipine & In favor of combination & Elderly; mixed gender \\
\hline & & & LDL-C (one study): MD -39.83 mg/dL (95\% Cl -71.29, -8.37) & High CHD risk \\
\hline & & & & 12 weeks treatment \\
\hline & & & In favor of combination & \\
\hline & & & TG (one study): MD, -23.91 mg/dL (95\% Cl -35.89, -11.93) & \\
\hline \multirow[t]{6}{*}{$\begin{array}{l}\text { Blood } \\
\text { pressure }\end{array}$} & $\begin{array}{l}\text { Omega-3-fish oil } \\
(2 \mathrm{~g} / \text { day })+\end{array}$ & Statins & In favor of combination & $\begin{array}{l}\text { Mean age: } 44 \text { to } 53 \mathrm{y} \text {; } \\
\text { mixed gender }\end{array}$ \\
\hline & & & $\begin{array}{l}\text { Systolic blood pressure (one study): } \mathrm{MD},-8.50 \mathrm{mmHg} \\
(95 \% \mathrm{Cl}-16.3,-0.66)\end{array}$ & Mixed $\mathrm{CHD}$ risk \\
\hline & & & & 5 weeks treatment \\
\hline & & & $\begin{array}{l}\text { Systolic blood pressure (one study): median change from } \\
\text { baseline }-5.0 \text { versus }+0.3 \mathrm{mmHg}\end{array}$ & \\
\hline & & & No difference between combination and CV drug alone & \\
\hline & & & $\begin{array}{l}\text { Diastolic blood pressure (one study): } \mathrm{MD}, 0.20 \mathrm{mmHg} \\
(95 \% \mathrm{Cl}-4.76,5.16)\end{array}$ & \\
\hline
\end{tabular}


Table 2 Gradable intermediate outcomes for dietary supplements plus cardiovascular drugs (low grade evidence) (Continued)

\begin{tabular}{lll}
\hline $\begin{array}{l}\text { Omega-3-fish oil } \\
(4 \mathrm{~g} / \text { day fish oil })+\end{array}$ & Statins & $\begin{array}{l}\text { Diastolic blood pressure (one study): Median reductions from } \\
\text { baseline }-3.30 \text { versus }-1.80 \text { to }\end{array}$ \\
\hline & \\
\hline
\end{tabular}

${ }^{a}$ Both studies recruited participant with higher baseline levels of triglyceride (>200 mg/dL). CHD: coronary heart disease; Cl: confidence interval; $C V$ : cardiovascular; $H D L-C$ : high density lipoprotein-cholesterol; $L D L$-cholesterol: low density lipoprotein-C; $M D$ : mean difference (post-treatment values); $R R$ : relative risk; $T G$ : triglycerides.

\section{Pharmacokinetic outcomes}

Results of one study showed no clinically important but a statistically significant decrease (grade: insufficient) in $\mathrm{S}$-warfarin $\mathrm{AUC}_{\infty}$, an increase in drug clearance and an apparent increase in volume of distribution (GMRs: 0.92 (90\% CI 0.85, 0.99); 1.09 (90\% CI 1.01, 1.18); and 1.09 (95\% CI 1.03, 1.18), respectively) [97].

\section{Garlic}

\section{Clinical outcome efficacy}

No evidence.

\section{Intermediate outcome efficacy}

In one study [61], a combination of garlic (4 g/day) and nitrates improved mean levels of HDL-C compared with nitrate alone (grade: low). Three other studies provided insufficient evidence for a combination of garlic plus warfarin, nitrates, or statin in addition to aspirin [60-62].

\section{Harms}

Evidence in four RCTs for garlic co-administered with warfarin, nitrates, or statins plus aspirin in healthy men [60] or those with CV conditions was inconclusive (grade: insufficient) $[59,61,62]$.

\section{Pharmacokinetic outcomes}

Evidence from two studies demonstrated neither clinically important (AUC, half-life or clearance; grade: low) [60] nor statistically significant $\left(C_{\max }\right.$; grade: insufficient) [102] interactions between garlic extracts and warfarin.

\section{Ginkgo biloba}

\section{Clinical outcome efficacy}

Insufficient evidence was found for the effect of Ginkgo biloba plus antiplatelets (aspirin and/or pentoxifylline) on all-cause mortality (no deaths in either group) in South Asians with previous stroke [47].

\section{Intermediate outcome efficacy}

Evidence from five RCTs for the combination of Ginkgo and acetylsalicylic acid, clopidogrel, ticlopidine, warfarin or cilostazol was inconclusive (grade: insufficient) [63-67].

\section{Harms}

Seven studies provided insufficient evidence for harm for the co-administration with warfarin, digoxin, aspirin, aspirin and/or pentoxifylline, nitrates, cilostazol or clopidogrel, or ticlopidine [47,63-67,98].

\section{Pharmacokinetic outcomes}

Three studies examined pharmacokinetic interaction of Ginkgo biloba and specific CV drugs (ticlopidine, digoxin and warfarin) $[63,66,98]$. Study results for clearance, AUC, half-life, or $\mathrm{C}_{\max }$ were either clinically not important or statistically non-significant (grade: insufficient).

\section{Omega-3 fatty acids \\ Clinical outcome efficacy}

Evidence on all-cause mortality for healthy individuals and highly selected patients with dyslipidemia taking statins as an add-on therapy [47], and patients with unclear to high 10-year CHD risk taking aspirin, warfarin or fenofibrate [55], was inconclusive (grade: insufficient). Similarly, evidence on acute myocardial infarction in high-risk patients taking a calcium channel antagonist was inconclusive [50]. Evidence from two studies on the incidence of restenosis following successful coronary angioplasty in patients taking omega- 3 fatty acids as an add-on to antiplatelet and calcium channel antagonist therapies was conflicting. One study showed significantly lower rates in the combination group (RR 0.40, 95\% CI $0.20,0.82$ ) [51] and the other study reported no significant difference between the groups (RR 1.33, 95\% CI $0.76,2.30)$ [50].

There is inconsistent evidence for a benefit when omega-3 fatty acids were added to conventional antiplatelet therapy and calcium channel antagonists on rates of acute myocardial infarction [50,51]. In three short-term RCTs $[38,43,52]$, adherence to CV drugs as judged by pill count was greater than $95 \%$ in both treatment groups.

\section{Intermediate outcome efficacy}

Co-administration with statins showed no benefit of lowering low density lipoprotein-cholesterol (LDL-C) (Figure 2) $[41,43,52,74,81]$, total cholesterol $[41,43,52,74,81]$, or of increasing HDL-C (grade: low) (Figure 3) $[41,43,52,74,75,81]$. However, the effect of adding statins 
Table 3 Gradable intermediate outcomes for dietary supplements plus cardiovascular drugs (insufficient grade evidence) ${ }^{a}$

\begin{tabular}{|c|c|c|c|}
\hline Item & Supplement (dose) & $\begin{array}{l}\text { Cardiovascular } \\
\text { drug }\end{array}$ & $\begin{array}{l}\text { Number of studies, sample size, characteristic } \\
\text { (treatment duration) }\end{array}$ \\
\hline \multirow{13}{*}{$\begin{array}{l}\text { All lipids (Low and } \\
\text { high density } \\
\text { lipoproteins-cholesterol, } \\
\text { triglycerides, total } \\
\text { cholesterol) } \\
\end{array}$} & $\begin{array}{l}\text { Coenzyme Q10 } \\
\text { (100 to } 200 \text { mg/day) }\end{array}$ & Statins & $\begin{array}{l}\text { Two studies; } 49 \text { hypercholesterolemic patients [57], and } \\
44 \text { patients with statin-induced myopathic pain [39]; (12 weeks) }\end{array}$ \\
\hline & Coenzyme Q10 (200 mg/day) & Fenofibrate & Participants with type II diabetes and high CHD risk \\
\hline & Garlic (4 g/day) & Warfarin & $\begin{array}{l}\text { Single study [59]; } 48 \text { participants with prosthetic heart valves, } \\
\text { or diagnosed with deep vein thrombosis, valvular heart disease } \\
\text { or atrial fibrillation }\end{array}$ \\
\hline & Garlic (4 mL/day) & Statins + aspirin & $\begin{array}{l}\text { Single study [62]; } 23 \text { participants with, or at high risk for, } \\
\text { coronary artery disease (1 year) }\end{array}$ \\
\hline & Garlic (4 g/day) & Nitrates & Single study [61]; 60 participants with coronary \\
\hline & & & artery disease (1 year) \\
\hline & Ginkgo biloba (120 mg/day) & Aspirin & Single study [64]; 50 young healthy men (1 week) \\
\hline & Omega-3 fatty acids (4 g/day) & Fenofibrate & Single study [55]; 167 participants with unclear CHD risk (8 weeks) \\
\hline & Omega-3 fatty acids (3 g/day) & $\begin{array}{l}\text { Calcium channel } \\
\text { blockers }\end{array}$ & Single study [49], 22 participants with variant angina (16 weeks) \\
\hline & Omega-3 fatty acids (4 g/day) & Niacin + aspirin & $\begin{array}{l}\text { Single study [77]; } 14 \text { participants with atherogenic dyslipidemia } \\
\text { (12 weeks) }\end{array}$ \\
\hline & Omega-3 fatty acids (10 g/day) & Aspirin & Two studies $[80,84] ; 30$ healthy participants (2 to 3 weeks) \\
\hline & Vitamin E (0.6/day) & Gemfibrozil & Single study [88]; 67 participants with hyperlipidemia (4 weeks) \\
\hline & Vitamin E (100 mg/day, 100 IU/day) & Statins & $\begin{array}{l}\text { Pooled results for four studies[89,91-93]; } 192 \text { highly selective } \\
\text { participants ( } 24 \text { weeks) }\end{array}$ \\
\hline \multirow[t]{3}{*}{ Triglycerides } & Omega-3 fatty acids (4 g/day) & ACE inhibitors & $\begin{array}{l}\text { Two studies }[78,79] ; 58 \text { participant with renal dysfunction or } \\
\text { hypertension ( } 6 \text { to } 25 \text { weeks) }\end{array}$ \\
\hline & Omega-3 fatty acids (4 to $9 \mathrm{~g} /$ day) & Statins & $\begin{array}{l}\text { Three studies [38,72,82]; } 420 \text { highly selected participants with } \\
\text { low or unclear CHD risk (4 to } 18 \text { weeks) }\end{array}$ \\
\hline & Vitamin E (900 mg/day) & Antiplatelet agents & Single study [86]; 16 participants with high CHD risk (6 weeks) \\
\hline $\begin{array}{l}\text { Low density } \\
\text { lipoprotein-cholesterol }\end{array}$ & Omega-3-fish oil (1.8 g/day) & $\begin{array}{l}\text { Calcium channel } \\
\text { blockers + aspirin }\end{array}$ & $\begin{array}{l}\text { Single study [50]; } 107 \text { participants with pre-coronary angioplasty } \\
\text { (6 weeks) }\end{array}$ \\
\hline \multirow{3}{*}{$\begin{array}{l}\text { High density } \\
\text { lipoprotein-cholesterol }\end{array}$} & Vitamin E (900 mg/day) & Nifedipine & Single study [94]; 30 participants with high CHD risk (16 weeks) \\
\hline & Omega-3 fatty acids ( $1.8 \mathrm{~g} /$ day $)$ & $\begin{array}{l}\text { Calcium channel } \\
\text { blockers + aspirin }\end{array}$ & $\begin{array}{l}\text { Single study [50]; } 107 \text { participants with pre-coronary angioplasty } \\
\text { (6 weeks) }\end{array}$ \\
\hline & Omega-3 fatty acids (3.2 g/day) & $\begin{array}{l}\text { Calcium channel } \\
\text { blockers + aspirin + } \\
\text { dipyridamole }\end{array}$ & $\begin{array}{l}\text { Single study [51]; } 82 \text { participants with post-coronary angioplasty } \\
\text { (12 weeks) }\end{array}$ \\
\hline \multirow[t]{9}{*}{$\begin{array}{l}\text { Blood pressure } \\
\text { (systolic and diastolic) }\end{array}$} & Coenzyme Q10 (200 mg/day) & Fenofibrate & $\begin{array}{l}\text { Single study[56]; } 80 \text { participants with type II diabetes and high } \\
\text { CHD risk (12 weeks) }\end{array}$ \\
\hline & Garlic (4 g/day) & Warfarin & $\begin{array}{l}\text { Single study [59]; } 48 \text { participants with prosthetic heart valves, } \\
\text { or diagnosed with deep vein thrombosis, valvular heart disease } \\
\text { or atrial fibrillation }\end{array}$ \\
\hline & Ginkgo biloba (120 mg/day) & Aspirin & Single study [64]; 50 young healthy male volunteers (1 week) \\
\hline & Ginkgo biloba (120 mg/day) & Cilostazol & Single study [67]; 10 healthy South Asian men (1 day) \\
\hline & Omega-3 fatty acids (10 g/day) & Aspirin & Two studies $[80,84] ; 30$ healthy participants (2 to 3 weeks) \\
\hline & Omega-3 fatty acids (4 g/day) & $\begin{array}{l}\text { Beta-adrenergic } \\
\text { antagonists }\end{array}$ & Single study [85]; 25 participants with unclear CHD risk (6 weeks) \\
\hline & Vitamin E (600 mg/day) & Furosemide & Single study [87]; 24 hypertensive participants (4 weeks) \\
\hline & & Gemfibrozil & Single study [88]; 67 participants with hyperlipidemia (4 weeks) \\
\hline & Vitamin E (900 mg/day) & Nifedipine & Single study [94]; 30 participants with high CHD risk (16 weeks) \\
\hline $\begin{array}{l}\text { International } \\
\text { normalized ratio }\end{array}$ & Echinacea (5 g/day) & Warfarin & Single study [97]; 12 healthy volunteers (2 weeks) \\
\hline
\end{tabular}


Table 3 Gradable intermediate outcomes for dietary supplements plus cardiovascular drugs (insufficient grade evidence) ${ }^{\mathbf{a}}$ (Continued)

\begin{tabular}{ll}
\hline Garlic (4 g/day) & $\begin{array}{l}\text { Two studies; 48 participants with high CHD risk [59] } \\
\text { (12 weeks), and 16 healthy men with known CYP2C9 and } \\
\text { VKORC1 genotype [60] (2 weeks) }\end{array}$ \\
\hline Ginger (3.6 g/day) & Single study [63];12 healthy male volunteers (7 days) \\
\hline Ginkgo biloba (12 g/day) & $\begin{array}{l}\text { Two studies; seven healthy men [70] (1 week), 25 patients with } \\
\text { ischemic stroke [68] (2 weeks) }\end{array}$ \\
\hline Panax ginseng (1.5-2 g/day) & Single study [54]; 11 participants with unclear CHD risk (4 weeks) \\
\hline Omega-3-fish oil (4 mg/day)
\end{tabular}

Inconclusive results: studies had an imprecise statistically non-significant pooled estimate (the $95 \%$ Cls were wide enough to be compatible with either clinical benefit, true no difference or harm). ACE: angiotensin-converting enzyme; CHD: coronary heart disease.

to omega-3 fatty acids in participants with high baseline triglyceride levels $(>200 \mathrm{mg} / \mathrm{mL})$ was beneficial in lowering post-treatment triglyceride levels [41,73] (grade: low), as opposed to participants with lower baseline levels of triglyceride for whom the results were inconclusive (grade: insufficient) $[41,43,52,73,74,81]$. Significant benefits in reducing systolic blood pressure (grade: low) $[38,76]$, but no difference in diastolic blood pressure (grade: low) $[74,76]$ were observed (Table 2).

\section{Harms}

Insufficient evidence was available from 22 studies (21 RCTs and one retrospective cohort study) which examined omega-3 fatty acids plus statins [37,38,41,52,72,76,81-
83,96,103], aspirin [46,51,77,80,84], aspirin and clopidogrel [99], aspirin in combination with dipyridamole and calcium channel blockers [51], warfarin [46,54], ramipril and/or irbesartan [78], or fenofibrate [55]. These studies were generally small and underpowered. They recruited healthy participants or participants with $\mathrm{CHD}$ or risk factors for CHD.

Meta-analyses of studies comparing omega-3 fatty acids and statin combinations versus statins alone yielded inconclusive results for serious adverse events (two studies; RR, 3.64 (95\% CI 0.8, 17.2)), withdrawal due to adverse events (seven studies; RR, 1.2 (95\% CI $0.6,2.3)$ ), elevated aspartate aminotransferase (two studies; RR, $0.6(95 \%$ CI $0.3,1.3))$ and elevated alanine transaminase (four studies; RR, 0.9 (95\% CI 0.5, 1.9)) (grade:

\section{LDL-C}

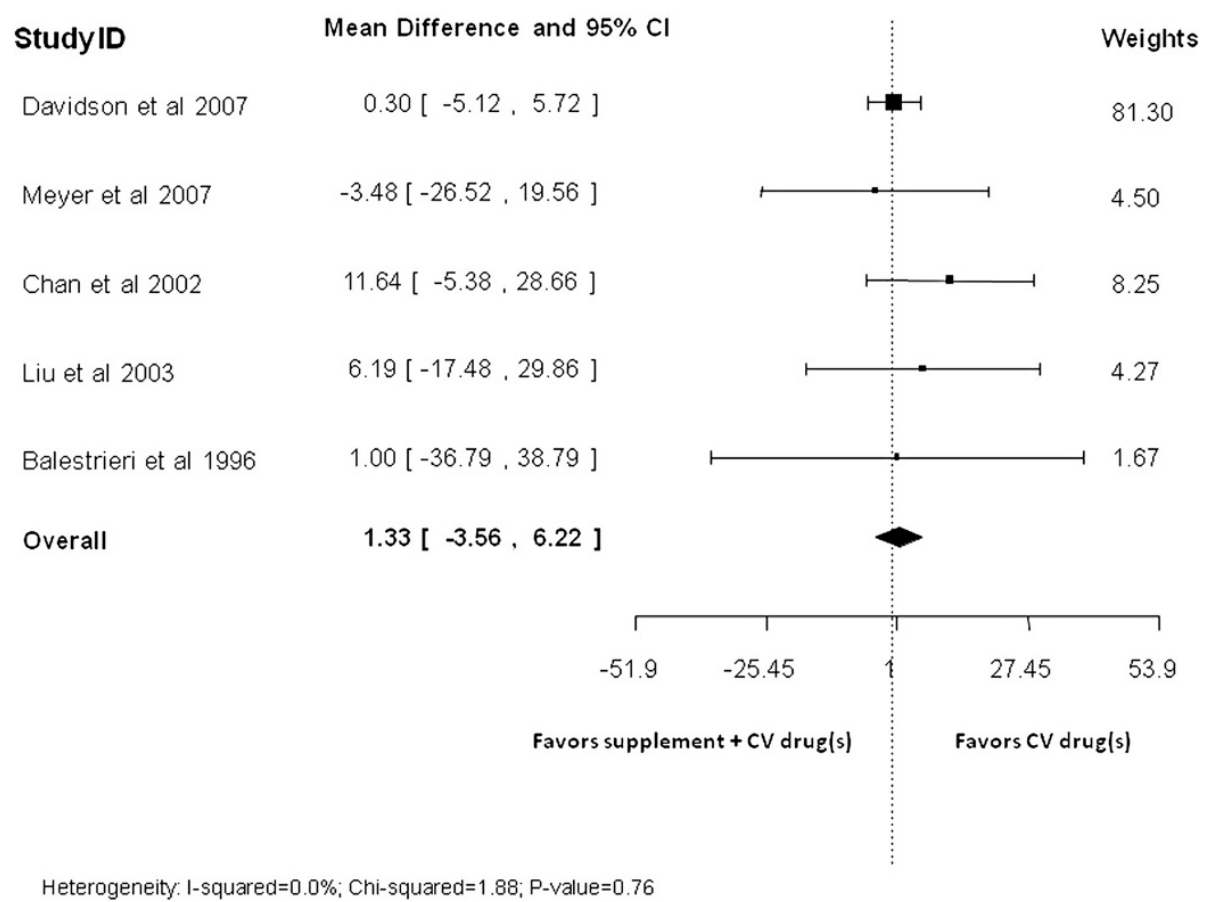

Figure 2 Omega-3 fatty acids co-administration with statins versus statins alone: post-treatment low density lipoprotein-cholesterol levels 


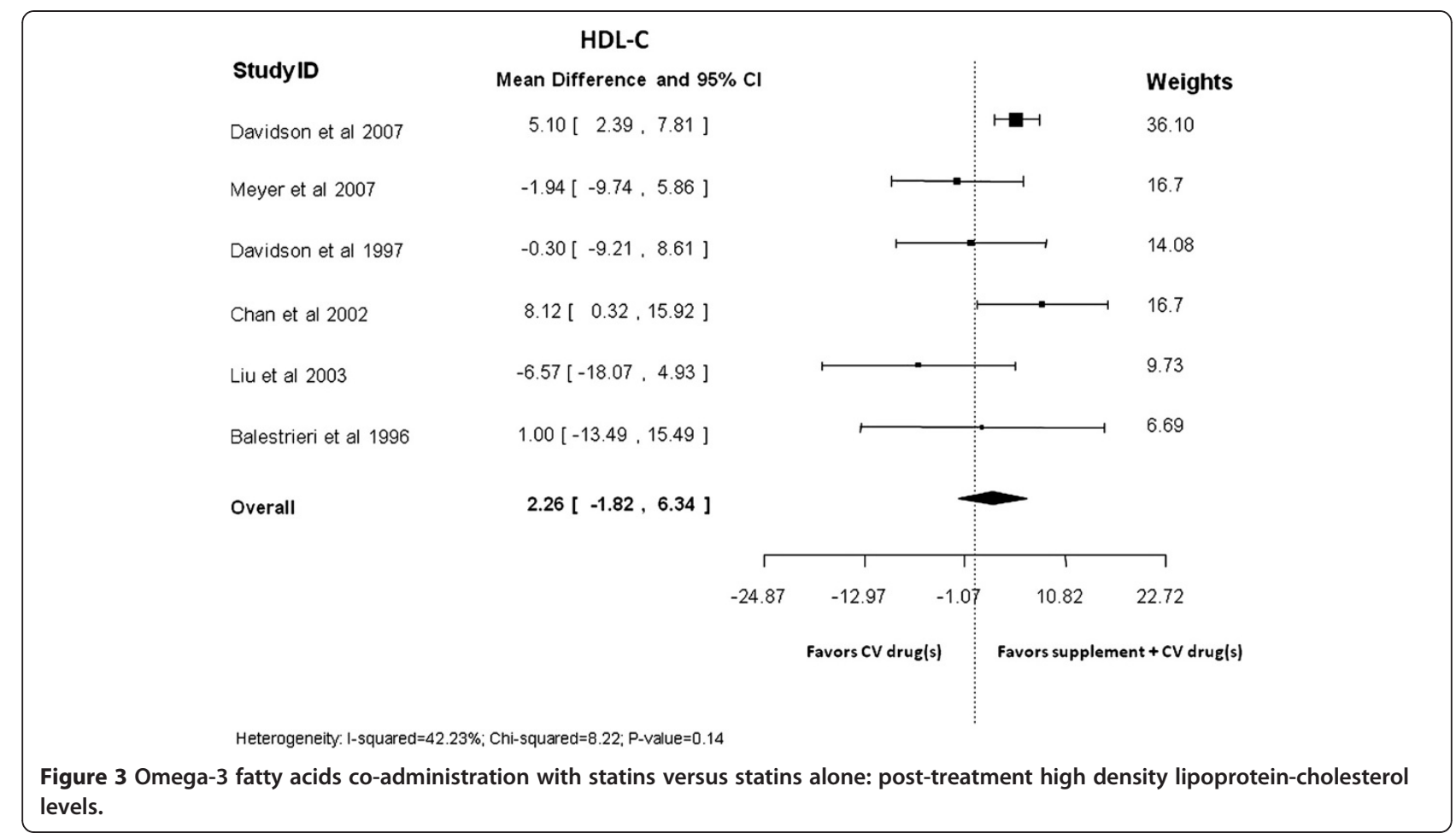

insufficient) [37,38,41,72,81,83,96]. Non-significant and imprecise pooled estimates were also obtained for total adverse events and elevated creatine kinase (grade: insufficient). Forest plots are available online [100].

\section{Pharmacokinetic outcomes}

In three open-label RCTs of healthy adult volunteers (sample size range 24 to 50 ) $[37,83,96]$ taking 4 g/day omega-3 fatty acids and rosuvastatin, atorvastatin, 2-hydroxy atorvastatin, or 4-hydroxy atorvastatin, no statistically significant or clinically important differences were observed between the treatment and control groups for steady state AUC and $C_{\max }$ GMRs (grade: low). Observed changes in steady state $\beta$-hydroxysimvastatin arithmetic means of AUC, $C_{\max }, t_{\max }$ clearance and halflife were not statistically significant (grade: insufficient).

\section{Vitamin E}

\section{Clinical outcome efficacy}

The evidence for the effect of vitamin $\mathrm{E}$ plus aspirin on stroke and transient ischemic attacks in selected patients with previous neurologic deficit was inconclusive (grade: insufficient) [48]. In one pragmatic trial, 19,934 women were randomized to vitamin E (600 IU/day) plus aspirin $(100 \mathrm{mg} /$ day) or aspirin alone for 10 years. No significant differences were noted for the composite outcome of nonfatal myocardial infarction, nonfatal stroke or vascular death (RR 0.95, 95\% CI 0.79, 1.13) [40]. Although components of the composite outcome were gradable, shifts in incidence of stroke and heart attack might have been obscured in this composite outcome. No differences in adherence to statins were noted with coadministration of vitamin $\mathrm{E}$.

Intermediate outcome efficacy

In one study of 30 elderly patients at high risk of CHD, addition of vitamin $\mathrm{E}$ to nifedipine significantly lowered total cholesterol, LDL-C (grade: low), triglycerides (grade: low) and systolic blood pressure (inconclusive, grade: insufficient) [94] (Tables 2, 3).

\section{Harms}

Evidence from 10 RCTs for co-administration of vitamin $\mathrm{E}$ and aspirin, nifedipine, furosemide, or statins in participants who were healthy, or who had CHD or risk factors for CHD was inconclusive (grade: insufficient). Sample sizes were generally small, except for one study that recruited over 9,000 women [40].

\section{Pharmacokinetic outcomes}

No evidence.

\section{Limitations of the results from the evidence}

Across all combinations of dietary supplements and CV drugs, the strength of evidence of the gradable outcomes of comparative efficacy or effectiveness was mostly graded as insufficient. Type II errors could not be excluded due to the low statistical power of mostly short- 
term efficacy trials, particularly with strict inclusion criteria excluding patients with uncontrolled comorbidities and acute ischemic events. In addition, most studies were short-term efficacy trials and thus unable to evaluate longer term effects from co-administration.

Limited findings on intermediate outcomes were available and the majority of evidence was contributed by small RCTs with statistically non-significant results and broad CIs. This imprecision precluded ruling in or out important benefits or harms, thus the strength of evidence for several gradable intermediate outcomes was rated insufficient. Statistically significant effects were graded as low strength of evidence because of limitations in the internal validity of studies, surrogacy of outcomes, and often absent reproducibility in the direction of effect estimates (Table 2). Forest plots are available online [100].

With respect to harms data, for all supplement-drug combinations examined, the strength of evidence from single or heterogeneous studies for bleeding, serious adverse events, withdrawal due to adverse events, renal dysfunction, hepatotoxicity and prolongation of corrected QT interval was insufficient. This was due to either inconsistent effect estimates across studies (suggesting conflicting findings with no obvious explanation) or imprecise estimates (precluding ruling out important benefit or harms in underpowered studies with wide confidence intervals).

\section{Discussion}

Evidence gleaned from an initial search yielding over 32,000 records identified a considerable knowledge gap regarding the safety and efficacy of combining dietary supplements with CV drugs.

Among 168 records that addressed the relevant possibility of interaction, 63 studies contributed evidence for synthesis. With a few exceptions, there was insufficient evidence to draw any conclusions on particular interactions. In addition to an overall lack of evidence, the included studies were often underpowered to assess the predetermined clinically relevant outcomes set for this synthesis review. As well, many studies had important methodological limitations or were poorly generalizable to the relevant population. The strength of the identified evidence was frequently compromised by poor allocation concealment, and issues related to blinding, study reporting and potential conflict of interest. Drug interactions resulting in positive or negative outcomes likely occur, but the evidence available and identified in this review is insufficient to allow meaningful conclusions with confidence.

Available evidence comes primarily from short-term trials of highly selected participants, with limited external validity. The strength of evidence was low at best, with poor grading resulting from risks of bias, small sample sizes, and the fact that evidence is largely generated from intermediate surrogate outcomes rather than primary clinical endpoints. While there are data from which we can derive a sense of lack of interactions in some cases, the small size of the trials made it difficult to impossible to ascertain the potential for true clinical interaction.

Much of the pharmacokinetic research was conducted on healthy young adults; thus this evidence may not be applicable to populations with CVD and particularly for older patients taking $\mathrm{CV}$ drugs, due to possible differences in metabolism and the existence of comorbidities. The internal validity of most trials was compromised by flawed design, lack of appropriate allocation concealment and risk of bias. A formal assessment of statistical interaction was rarely undertaken. In the absence of corroborating pharmacokinetic evidence or assessment for statistical interaction, it is often impossible to determine whether a difference in outcome is due to true pharmacological interaction, or due to more independent additive, or possibly counteracting therapeutic effects.

A principal limitation of the included trials was that they were small and susceptible to type II errors. A marginally reassuring corollary is that if there was a real, dramatic clinical impact due to an interaction then some clinical effect would likely have been evident despite being underpowered for smaller effect sizes. Of more concern are possible interactions that could arise through polypharmacy of prescription drugs, a situation all too common, in particular for the elderly population.

With these caveats in mind, the following is a summary of the clearer signals from the evidence reviewed.

Omega-3 fatty acids ( 2 to $4 \mathrm{~g} /$ day) from fish and/or supplements likely do not interfere with the efficacy of statin therapy or calcium channel blockers in the presence of antiplatelet agents, and may provide an independent benefit in resolving hypertriglyceridemia. Also, garlic (4 to $10 \mathrm{~g} /$ day) may not interact negatively with nitrates and warfarin and may confer independent benefit in improving HDL-C.

Interpretation of this report requires the reader to recognize that dietary supplements in the US are not regulated in the same manner as prescription drugs nor are manufacturers of dietary supplements held to the same standards with respect to providing evidence of efficacy and safety prior to marketing. Dietary supplements do not require FDA approval, nor are there any FDA regulations that require evidence of purity, quality or composition prior to marketing. This has resulted in a lack of standardization among products both from a single manufacturer and between manufacturers [104]. The lack of manufacturing regulation and labeling standards may result in significant differences between products, unbeknownst to the consumer, thereby limiting the external validity of clinical trials. Furthermore, there is little reliable published information regarding the safety of dietary supplements. Until only recently, 
manufacturers of dietary supplements were not obliged to report serious adverse events. This is quite different from what is required of prescription drugs.

Recent systematic reviews related to the topic of dietary supplement-drug interactions do not address the same scope, are not comprehensive, do not grade the outcomes extracted, do not evaluate the quality of evidence, or involve different populations of interest. A 2005 systematic review by Mills et al. focused on effects of natural health products on the metabolism of a broad range of conventional medicines [105]. Consistent with our review, they identified a lack of evidence supporting interactions between coenzyme Q10 and warfarin, Ginkgo biloba and warfarin, and Ginkgo biloba and digoxin. Both reviews also identified a shift in AUC of the international normalized ratio when American ginseng was taken in conjunction with warfarin; however, we question the clinical significance of this finding.

Also in 2005, Desai et al. reviewed interactions between dietary supplements and antiplatelet agents reported in clinical trials and case reports [106]. With respect to the supplements considered in our review, Desai concluded that omega-3 fatty acids along with aspirin led to significantly greater reductions in adenosine diphosphateinduced platelet aggregation, blood platelet count, thromboxane B2 and restenosis rates, as well as prolonged bleeding time. Vitamin $\mathrm{E}$ along with aspirin led to significantly greater reductions in platelet adhesion, ischemic stroke, recurrent episodes of transient ischemic attack, as well as prolonged dental bleeding time.

Reviews published by Izzo et al. in 2005 and Skalli et al. in 2007 described evidence for drug-supplement interactions, but the majority of evidence was generated from case reports and small case series $[107,108]$. The most recent systematic review on dietary supplementdrug interactions, published in 2010 by Kennedy and Seely, examined herb-drug interactions identified from trials wherein the herbal impact on hepatic metabolism via cytochrome P450 isoenzymes was ascertained [109]. Their target population was not specifically patients with CVD or CV drugs, and the review evaluated indirect evidence limited to herbs metabolized via the cytochrome P450 system. While some of the findings within these reviews are consistent with ours, our graded evaluation did not yield similar confidence in conclusions after evaluating the quality and strength of evidence.

A most obvious limitation of our review arose from the need to scope the work to a manageable yet relevant synthesis by restricting the number of dietary supplements to the sixteen that were considered. This subset of supplements was based on published North American usage surveys and a consensus selection process that included a Technical Experts Panel [6,21-26]. Thus, we omitted drugs and supplements, and related data from other international sources. The review also did not consider combinations of multiple dietary supplements with $\mathrm{CV}$ drugs to make causal inference possible. This limitation has negative implications for external validity because people with CVD likely selfprescribe combinations of numerous dietary supplements, taken alongside their $\mathrm{CV}$ drugs.

The greatest strengths of the present review are its broad search strategy and consideration of extensive literature, and a methodology that focused on the highest quality of evidence. We followed published guidance to pool data from studies, grade the strength of evidence, and assess applicability. Based upon preliminary searches we did not exclude German language literature, a language where most negative studies are published [110]. In order to minimize over-generalization of evidence, we also included only studies with more than $80 \%$ of participants taking each $\mathrm{CV}$ medication under consideration.

While a plethora of strong recommendations was the hope, we revealed instead a glaring knowledge gap regarding interactions between some of the agents most commonly used for their presumed pharmacological effects for serious chronic disease.

\section{Conclusion}

With the continuing and increasing burden of CVD, precise recommendations are needed to guide the use of dietary supplements in disease prevention and management. An ungrounded call for avoidance of dietary supplements in conjunction with pharmaceutical treatments may result in the avoidance of potentially beneficial supplements, or just as importantly might be readily ignored, leading to potential harm with negative interactions. Strategic investment to build research capacity to address the important knowledge gap in the area of dietary supplement-drug interactions should address relevant questions in appropriate populations according to disease, genetic makeup, age, and so on. While awaiting results of future, adequately powered interventional trials focusing on meaningful clinical outcomes, evidence from well-conducted prospective observational studies should be sought. Electronic health record linkages between databases of dietary supplement use and CV drug prescription may also add to the equipoise that has been so insufficiently addressed to date.

\section{Additional files}

Additional file 1: Table S1. Selected cardiovascular drugs for current review.

Additional file 2: Table S2. List of included outcomes.

Additional file 3: Table S3. a Priori outcomes for grading the strength of evidence. 


\section{Abbreviations}

AHRQ: Agency for Healthcare Research and Quality; AUC: area under the curve; Cl: confidence interval; CHD: coronary heart disease; CV: cardiovascular; CVD: cardiovascular disease; FDA: Food and Drug Administration; GMR: geometric mean ratio; HDL-C: high density lipoprotein-cholesterol; INR: International Normalized Ratio; LDL-C: low density lipoproteincholesterol; MD: mean difference; OR: odds ratios; RCT: randomized clinical trials; RR: relative risk

\section{Competing interests}

The authors declare that they have no competing interests.

\section{Authors' contribution}

SK and DS were involved in the conception and design, data interpretation, reviewing and revising the manuscript critically for important intellectual content, and its final approval. FY, KS, AT, ACT and MS analyzed and interpreted data, reviewed the draft and revised it critically for important intellectual content, and approved the manuscript. JT was involved in the conception and design, reviewed the draft and revised the manuscript critically for important intellectual content, and approved the manuscript. $\mathrm{TCO}, \mathrm{MAT}$ and BS were involved in the conception and design, reviewed the draft and revised it critically for important intellectual content, and approved the manuscript. MTA was involved in the conception and design, data analysis and interpretation, drafting of the manuscript, and final approval of the manuscript. All authors read and approved the final manuscript.

\section{Disclaimer}

This project was funded under Contract No. HHSA290-2007-10059-I (EPCIII) from the AHRQ, US Department of Health and Human Services. The authors of this report are responsible for its content. Statements in the report should not be construed as endorsement by the AHRQ, National Institute of Health or the US Department of Health and Human Services.

\section{Acknowledgments}

The authors gratefully acknowledge the following individuals for their contributions to this manuscript: Raymond Daniel, James Galipeau, Mary Gauthier, Mistrel Pratt and Sophia Tsouros assisted with article acquisition, reference generation, project management, screening, data extraction, development of the evidence tables, and preparation of the original evidence report; Katja Boehm and Stefania Milazzo assisted with screening of the German articles; Ranjeeta Mallick and Lucy Turner provided statistical support and consultations for data analysis.

\section{Author details}

${ }^{1}$ Clinical Epidemiology, The Ottawa Hospital Research Institute and the Department of Pharmacy, The Ottawa Hospital, Ottawa, ON, Canada. ${ }^{2}$ Department of Research \& Clinical Epidemiology, The Canadian College of Naturopathic Medicine, Toronto, ON, Canada. ${ }^{3}$ Ottawa Methods Centre, Clinical Epidemiology Program, Ottawa Hospital Research Institute, University of Ottawa Evidence-based Practice Center, Ottawa, ON, Canada. ${ }^{4}$ Li Ka Shing Knowledge Institute, St Michael's Hospital, Toronto, ON, Canada. ${ }^{5}$ Children's Hospital of Eastern Ontario Research Institute, Ottawa, ON, Canada. ${ }^{6}$ Division of Endocrinology and Metabolism, The Ottawa Hospital, University of Ottawa, Ottawa, ON, Canada. ${ }^{7}$ Division of Cardiology, The Ottawa Hospital, University of Ottawa, Ottawa ON, Canada.

Received: 6 December 2011 Accepted: 18 April 2012

Published: 31 May 2012

\section{References}

1. Lloyd-Jones D, Adams RJ, Brown TM, Carnethon M, Dai S, de Simone G, Ferguson TB, Ford E, Furie K, Gillespie C, Go A, Greenlund K, Haase N, Hailpern S, Ho PM, Howard V, Kissela B, Kittner S, Lackland D, Lisabeth L, Marelli A, McDermott MM, Meigs J, Mozaffarian D, Mussolino M, Nichol G, Roger VL, Rosamond W, Sacco R, Sorlie P, et al: Heart disease and stroke statistics-2010 update: a report from the American Heart Association. Circ Cardiovasc Qual Outcomes 2010, 121:e46-e215.

2. Brautbar A, Ballantyne CM: Pharmacological strategies for lowering LDL cholesterol: statins and beyond. Nat Rev Cardiol 2011, 8:253-265.
3. Mills EJ, Rachlis B, Wu P, Devereaux PJ, Arora P, Perri D: Primary prevention of cardiovascular mortality and events with statin treatments: a network meta-analysis involving more than 65,000 patients. J Am Coll Cardiol 2008, 52:1769-1781.

4. Choudhry NK, Fischer MA, Avorn J, Liberman JN, Schneeweiss S, Pakes J, Brennan TA, Shrank WH: The implications of therapeutic complexity on adherence to cardiovascular medications. Arch Intern Med 2011, 171:814-822.

5. Nahin RL, Barnes PM, Stussman BJ, Bloom B: Costs of complementary and alternative medicine (CAM) and frequency of visits to CAM practitioners: United States. Natl Health Stat Report 2007, 2009:1-14.

6. Yeh GY, Davis RB, Phillips RS: Use of complementary therapies in patients with cardiovascular disease. Am J Cardiol 2006, 98:673-680.

7. Zick SM, Blume A, Aaronson KD: The prevalence and pattern of complementary and alternative supplement use in individuals with chronic heart failure. J Card Fail 2005, 11:586-589.

8. Tachjian A, Maria V, Jahangir A: Use of herbal products and potential interactions in patients with cardiovascular diseases. J Am Coll Cardiol 2010, 55:515-525.

9. Mashour NH, Lin Gl, Frishman WH: Herbal medicine for the treatment of cardiovascular disease: clinical considerations. Arch Intern Med 1998, 158:2225-2234.

10. Maraldi C, Lattanzio F, Onder G, Gallerani M, Bustacchini S, De TG, Volpato S: Variability in the prescription of cardiovascular medications in older patients: correlates and potential explanations. Drugs Aging 2009, 26(Suppl 1):41-51.

11. Garner JB: Problems of nonadherence in cardiology and proposals to improve outcomes. Am J Cardiol 2010, 105:1495-1501.

12. Miller KL, Liebowitz RS, Newby LK: Complementary and alternative medicine in cardiovascular disease: a review of biologically based approaches. Am Heart J 2004, 147:401-411.

13. Ulbricht C, Chao W, Costa D, Rusie-Seamon E, Weissner W, Woods J: Clinical evidence of herb-drug interactions: a systematic review by the natural standard research collaboration. Curr Drug Metab 2008, 9:1063-1120.

14. The Agency for Healthcare Research and Quality. http://www. effectivehealthcare.ahrq.gov/index.cfm.

15. Wu T, Li Y, Bian Z, Liu G, Moher D: Randomized trials published in some Chinese journals: how many are randomized? Trials 2009, 10:46.

16. Shekelle PG, Morton SC, Suttorp MJ, Buscemi N, Friesen C: Challenges in systematic reviews of complementary and alternative medicine topics. Ann Intern Med 2005, 142:1042-1047.

17. Halsted $\mathrm{CH}$ : Dietary supplements and functional foods: 2 sides of a coin?. Am J Clin Nutr 2003, 77:1001S-1007S.

18. Coppens $P$, da Silva MF, Pettman S: European regulations on nutraceuticals, dietary supplements and functional foods: a framework based on safety. Toxicology 2006, 221:59-74.

19. Eberhardie C: Nutritional supplements and the EU: is anyone happy?. Proc Nutr Soc 2007, 66:508-511.

20. US Food and Drug Administration: Overview of Dietary Supplements. http://www.fda.gov/food/dietarysupplements/consumerinformation/ ucm110417.htm

21. Pharand C, Ackman ML, Jackevicius CA, Paradiso-Hardy FL, Pearson GJ: Canadian Cardiovascular Pharmacists Network: Use of OTC and herbal products in patients with cardiovascular disease. Ann Pharmacother 2003, 37:899-904.

22. Gohar F, Greenfield SM, Gareth BD, Lip GYH, Jolly K: Self-care and adherence to medication: a survey in the hypertension outpatient clinic. BMC Complement Altern Med 2008, 8:4.

23. Balluz LS, Kieszak SM, Philen RM, Mulinare J: Vitamin and mineral supplement use in the United States: results from the third national health and nutrition examination survey. Arch Fam Med 2000, 9:258-262.

24. Schellhorn B, Doring A, Stieber J: Use of vitamin and mineral supplements: Results from the survey 1994/95 of the WHO MONICA Project Augsburg. Z Ernährungswiss 1998, 37:198-206.

25. Nahin RL, Pecha M, Welmerink DB, Sink K, DeKosky ST, Fitzpatrick AL: Ginkgo Evaluation of Memory Study Investigators: Concomitant use of prescription drugs and dietary supplements in ambulatory elderly people. J Am Geriatr Soc 2009, 57:1197-1205.

26. Barnes PM, Bloom B, Nahin RL: Complementary and alternative medicine use among adults and children: United States. Natl Health Stat Report 2007, 2008:1-23. 
27. Grundy SM, Cleeman Jl, Merz CN, Brewer HB Jr, Clark LT, Hunninghake DB, Pasternak RC, Smith SC Jr, Stone NJ: Implications of recent clinical trials for the National Cholesterol Education Program Adult Treatment Panel III guidelines. Circ Cardiovasc Qual Outcomes 2004, 110:227-239.

28. Owens DK, Lohr KN, Atkins D, Treadwell JR, Reston JT, Bass EB, Chang S, Helfand M: AHRQ series paper 5: grading the strength of a body of evidence when comparing medical interventions-agency for healthcare research and quality and the effective health-care program. J Clin Epidemiol 2010, 63:513-523.

29. Atkins D, Chang SM, Gartlehner G, Buckley DI, Whitlock EP, Berliner E, Matchar D: Assessing applicability when comparing medical interventions: AHRQ and the Effective Health Care Program. J Clin Epidemiol 2011, 64:1198-1207.

30. DerSimonian R, Laird N: Meta-analysis in clinical trials. Control Clin Trials 1986, 7:177-188.

31. Fu R, Gartlehner G, Grant M, Shamliyan T, Sedrakyan A, Wilt TJ, Griffith L, Oremus M, Raina P, Ismaila A, Santaguida P, Lau J, Trikalinos TA: Conducting quantitative synthesis when comparing medical interventions: AHRQ and the effective health care program. J Clin Epidemiol 2011, 64(11):1187-1197.

32. US Food and Drugs Administration: Approved drug products with therapeutic equivalence evaluations. Silver Spring (MD): US Food and Drug Administration; 2011

33. Takkouche B, Etminan M, Caamano F, Rochon PA: Interaction between aspirin and ACE Inhibitors: resolving discrepancies using a meta-analysis. Drug Saf 2002, 25:373-378.

34. The Agency for Healthcare Research and Quality. Available at: http://www. effectivehealthcare.ahrq.gov/index.cfm. Last Accessed: 8-3-2012.

35. Borenstein M, Hedges L, Higgins J, Rothstein H: Comprehensive meta analysis. Englewood NJ: 2005. Version 2.2.057.

36. StatsDirect Ltd: StatsDirect statistical software. UK: Altrincham; 2012.

37. Di Spirito M, Morelli G, Doyle RT, Johnson J, McKenney J: Effect of omega3-acid ethyl esters on steady-state plasma pharmacokinetics of atorvastatin in healthy adults. Expert Opin Pharmacother 2008, 9:2939-2945.

38. Maki KC, McKenney JM, Reeves MS, Lubin BC, Dicklin MR: Effects of adding prescription omega-3 acid ethyl esters to simvastatin ( $20 \mathrm{mg} /$ day) on lipids and lipoprotein particles in men and women with mixed dyslipidemia. Am J Cardiol 2008, 102:429-433. Erratum appears in Am J Cardiol 2008; 102(10):1425

39. Young JM, Florkowski CM, Molyneux SL, McEwan RG, Frampton CM, George PM, Scott RS: Effect of coenzyme Q(10) supplementation on simvastatininduced myalgia. Am J Cardiol 2007, 100:1400-1403.

40. Glynn RJ, Ridker PM, Goldhaber SZ, Zee RY, Buring JE: Effects of random allocation to vitamin $E$ supplementation on the occurrence of venous thromboembolism: report from the Women's Health Study. Circ Cardiovasc Qual Outcomes 2007, 116:1497-1503.

41. Davidson MH, Stein EA, Bays HE, Maki KC, Doyle RT, Shalwitz RA, Ballantyne CM, Ginsberg HN: COMBination of prescription Omega-3 with Simvastatin (COMBOS) Investigators: Efficacy and tolerability of adding prescription omega-3 fatty acids $4 \mathrm{~g} / \mathrm{d}$ to simvastatin $40 \mathrm{mg} / \mathrm{d}$ in hypertriglyceridemic patients: an 8-week, randomized, double-blind, placebo-controlled study. Clin Ther 2007, 29:1354-1367.

42. Miyamoto S, Kawano H, Takazoe K, Soejima H, Sakamoto T, Hokamaki J, Yoshimura M, Nakamura H, Yodoi J, Ogawa H: Vitamin E improves fibrinolytic activity in patients with coronary spastic angina. Thromb Res 2004, 113:345-351.

43. Chan DC, Watts GF, Mori TA, Barrett PH, Beilin LJ, Redgrave TG: Factorial study of the effects of atorvastatin and fish oil on dyslipidaemia in visceral obesity. Eur J Clin Invest 2002, 32:429-436.

44. Motoyama T, Kawano H, Kugiyama K, Hirashima O, Ohgushi M, Tsunoda R, Moriyama Y, Miyao Y, Yoshimura M, Ogawa H, Yasue H: Vitamin E administration improves impairment of endothelium-dependent vasodilation in patients with coronary spastic angina. J Am Coll Cardiol 1998, 32:1672-1679.

45. Napoli C, Leccese M, Palumbo G, De NF, Chiariello P, Zuliani P, Somma P, Di LM, De MC, Cacciatore F, Abete P, Liguori A, Chiariello M, D'Armiento FP. Effects of vitamin $\mathrm{E}$ and $\mathrm{HMG}-\mathrm{CoA}$ reductase inhibition on cholesteryl ester transfer protein and lecithin-cholesterol acyltransferase in hypercholesterolemia. Coron Artery Dis 1998, 9:257-264.
46. Eritsland J, Arnesen H, Nseth K, Fjeld NB, Abdelnoor M: Effect of dietary supplementation with $\mathrm{n}-3$ fatty acids on coronary artery bypass graft patency. Am J Cardiol 1996, 77:31-36

47. Garg RK, Nag D, Agrawal A: A double blind placebo controlled trial of ginkgo biloba extract in acute cerebral ischaemia. J Assoc Phys India 1995, 43:760-763.

48. Steiner M, Glantz M, Lekos A: Vitamin E plus aspirin compared with aspirin alone in patients with transient ischemic attacks. Am J Clin Nutr 1995, 62:1381S-1384S.

49. Yamamoto H, Yoshimura H, Noma M, Suzuki S, Kai H, Tajimi T, Sugihara M, Kikuchi Y: Improvement of coronary vasomotion with eicosapentaenoic acid does not inhibit acetylcholine-induced coronary vasospasm in patients with variant angina. Jpn Circ J 1995, 59:608-616. Erratum appears in Jpn Circ J 1995, 59(11):773-774.

50. Kaul U, Sanghvi S, Bahl VK, Dev V, Wasir HS: Fish oil supplements for prevention of restenosis after coronary angioplasty. Int J Cardiol 1992, 35:87-93

51. Dehmer GJ, Popma JJ, van den Berg EK, Eichhorn EJ, Prewitt JB, Campbell WB, Jennings L, Willerson JT, Schmitz JM: Reduction in the rate of early restenosis after coronary angioplasty by a diet supplemented with n-3 fatty acids. N Engl J Med 1988, 319:733-740.

52. Liu M, Wallmon A, Wallin R, Saldeen T: Effects of stable fish oil and simvastatin on plasma lipoproteins in patients with hyperlipidemia. Nutr Res 2003, 23:1027-1034.

53. Watson PS, Scalia GM, Galbraith A, Burstow DJ, Bett N, Aroney CN: Lack of effect of coenzyme $Q$ on left ventricular function in patients with congestive heart failure. J Am Coll Cardiol 1999, 33:1549-1552.

54. Bender NK, Kraynak MA, Chiquette E, Linn WD, Clark GM, Bussey HI: Effects of marine fish oils on the anticoagulation status of patients receiving chronic warfarin therapy. J Thromb Thrombolysis 1998, 5:257-261.

55. Roth EM, Bays HE, Forker AD, Maki KC, Carter R, Doyle RT, Stein EA: Prescription omega-3 fatty acid as an adjunct to fenofibrate therapy in hypertriglyceridemic subjects. J Cardiovasc Pharmacol 2009, 54:196-203.

56. Playford DA, Watts GF, Croft KD, Burke V: Combined effect of coenzyme Q10 and fenofibrate on forearm microcirculatory function in type 2 diabetes. Atherosclerosis 2003, 168:169-179.

57. Mabuchi H, Nohara A, Kobayashi J, Kawashiri MA, Katsuda S, Inazu A, Koizumi J, Hokuriku Lipid Research Group: Effects of CoQ10 supplementation on plasma lipoprotein lipid, CoQ10 and liver and muscle enzyme levels in hypercholesterolemic patients treated with atorvastatin: a randomized double-blind study. Atherosclerosis 2007, 195:e182-e189.

58. Caso G, Kelly P, McNurlan MA, Lawson WE: Effect of coenzyme q10 on myopathic symptoms in patients treated with statins. Am J Cardiol 2007, 99:1409-1412.

59. Macan H, Uykimpang $R$, Alconcel M, Takasu J, Razon R, Amagase H, Niihara $Y$ : Aged garlic extract may be safe for patients on warfarin therapy. J Nutr 2006, 136:793S-795S.

60. Mohammed Abdul MI, Jiang X, Williams KM, Day RO, Roufogalis BD, Liauw WS, Xu H, McLachlan AJ: Pharmacodynamic interaction of warfarin with cranberry but not with garlic in healthy subjects. $\mathrm{Br} J$ Pharmacol 2008 , 154:1691-1700.

61. Bordia A, Verma SK, Srivastava KC: Effect of garlic (Allium sativum) on blood lipids, blood sugar, fibrinogen and fibrinolytic activity in patients with coronary artery disease. Prostaglandins Leukot Essent Fatty Acids 1998, 58:257-263.

62. Budoff MJ, Takasu J, Flores FR, Niihara Y, Lu B, Lau BH, Rosen RT, Amagase $\mathrm{H}$ : Inhibiting progression of coronary calcification using Aged Garlic Extract in patients receiving statin therapy: a preliminary study. Prev Med 2004, 39:985-991.

63. Jiang X, Williams KM, Liauw WS, Ammit AJ, Roufogalis BD, Duke CC, Day RO, McLachlan AJ: Effect of ginkgo and ginger on the pharmacokinetics and pharmacodynamics of warfarin in healthy subjects. Br J Clin Pharmacol 2005, 59:425-432.

64. Wolf HR: Does Ginkgo biloba special extract EGb 761 provide additional effects on coagulation and bleeding when added to acetylsalicylic acid $500 \mathrm{mg}$ daily?. Drugs in R D 2006, 7:163-172.

65. Gardner CD, Zehnder JL, Rigby AJ, Nicholus JR, Farquhar JW: Effect of Ginkgo biloba (EGb 761) and aspirin on platelet aggregation and platelet function analysis among older adults at risk of cardiovascular disease: a randomized clinical trial. Blood Coagul Fibrinolysis 2007, 18:787-793. 
66. Kim BH, Kim KP, Lim KS, Kim JR, Yoon SH, Cho JY, Lee YO, Lee KH, Jang IJ, Shin SG, Yu KS: Influence of Ginkgo biloba extract on the pharmacodynamic effects and pharmacokinetic properties of ticlopidine: an open-label, randomized, two-period, two-treatment, two-sequence, single-dose crossover study in healthy Korean male volunteers. Clin Ther 2010, 32:380-390.

67. Aruna D, Naidu MU: Pharmacodynamic interaction studies of Ginkgo biloba with cilostazol and clopidogrel in healthy human subjects. $\mathrm{Br} J$ Clin Pharmacol 2007, 63:333-338.

68. Lee SH, Ahn YM, Ahn SY, Doo HK, Lee BC: Interaction between warfarin and Panax ginseng in ischemic stroke patients. J Altern Complement Med 2008, 14:715-721.

69. Yuan CS, Wei G, Dey L, Karrison T, Nahlik L, Maleckar S, Kasza K, Ang-Lee M, Moss J: Brief communication: American ginseng reduces warfarin's effect in healthy patients: a randomized, controlled trial. Ann Intern Med 2004, 141:23-27.

70. Jiang X, Williams KM, Liauw WS, Ammit AJ, Roufogalis BD, Duke CC, Day RO, McLachlan AJ: Effect of St John's wort and ginseng on the pharmacokinetics and pharmacodynamics of warfarin in healthy subjects. Br J Clin Pharmacol 2004, 57:592-599. [Erratum appears in Br J Clin Pharmacol 2004, 58(1):102.

71. Tankanow R, Tamer HR, Streetman DS, Smith SG, Welton JL, Annesley T, Aaronson KD, Bleske BE: Interaction study between digoxin and a preparation of hawthorn (Crataegus oxyacantha). J Clin Pharmacol 2003, 43:637-642.

72. Bays HE, McKenney J, Maki KC, Doyle RT, Carter RN, Stein E: Effects of prescription omega-3-acid ethyl esters on non-high-density lipoprotein cholesterol when coadministered with escalating doses of atorvastatin. Mayo Clin Proc 2010, 85:122-128.

73. Nordøy A, Bonaa KH, Sandset PM, Hansen JB, Nilsen H: Effect of omega-3 fatty acids and simvastatin on hemostatic risk factors and postprandial hyperlipemia in patients with combined hyperlipemia. Arterioscler Thromb Vasc Biol 2000, 20:259-265.

74. Meyer BJ, Hammervold T, Rustan AC, Howe PR: Dose-dependent effects of docosahexaenoic acid supplementation on blood lipids in statin-treated hyperlipidaemic subjects. Lipids 2007, 42:109-115.

75. Davidson MH, Macariola-Coad JR, McDonald AM, Maki KC, Hall HA: Separate and joint effects of marine oil and simvastatin in patients with combined hyperlipidemia. Am J Cardiol 1997, 80:797-798.

76. Nordøy A, Svensson B, Hansen JB: Atorvastatin and omega-3 fatty acids protect against activation of the coagulation system in patients with combined hyperlipemia. J Thromb Haemost 2003, 1:690-697.

77. Isley WL, Miles JM, Harris WS: Pilot study of combined therapy with omega-3 fatty acids and niacin in atherogenic dyslipidemia. J Clin Lipidol 2007, 1:211-217.

78. Ferraro PM, Ferraccioli GF, Gambaro G, Fulignati P, Costanzi S: Combined treatment with renin-angiotensin system blockers and polyunsaturated fatty acids in proteinuric IgA nephropathy: a randomized controlled trial. Nephrol Dial Transplant 2009, 24:156-160.

79. Howe PR, Lungershausen YK, Cobiac L, Dandy G, Nestel PJ: Effect of sodium restriction and fish oil supplementation on BP and thrombotic risk factors in patients treated with ACE inhibitors. J Hum Hypertens 1994, 8:43-49.

80. Svaneborg N, Kristensen SD, Hansen LM, Llow I, Husted SE, Schmidt EB: The acute and short-time effect of supplementation with the combination of $\mathrm{n}-3$ fatty acids and acetylsalicylic acid on platelet function and plasma lipids. Thromb Res 2002, 105:311-316.

81. Balestrieri GP, Maffi V, Sleiman I, Spandrio S, Di SO, Salvi A, Scalvini T: Fish oil supplementation in patients with heterozygous familial hypercholesterolemia. Recenti Prog Med 1996, 87:102-105.

82. Hansen JB, Lyngmo V, Svensson B, Nord YA: Inhibition of exercise-induced shortening of bleeding time by fish oil in familial hypercholesterolemia (type Ila). Rterioscler Thromb 1993, 13:98-104.

83. McKenney JM, Swearingen D, Di SM, Doyle R, Pantaleon C, Kling D, Shalwitz RA: Study of the pharmacokinetic interaction between simvastatin and prescription omega-3-acid ethyl esters. J Clin Pharmacol 2006, 46:785-791.

84. Mueller BA, Talbert RL, Tegeler $\mathrm{CH}$, Prihoda TJ: The bleeding time effects of a single dose of aspirin in subjects receiving omega-3 fatty acid dietary supplementation. J Clin Pharmacol 1991, 31:185-190.
85. Lungershausen YK, Howe PR: Improved detection of a blood pressure response to dietary intervention with 24-hour ambulatory monitoring. Am J Hypertens 1994, 7:1115-1117.

86. Micheletta F, Natoli S, Misuraca M, Sbarigia E, Diczfalusy U, Iuliano L: Vitamin E supplementation in patients with carotid atherosclerosis: reversal of altered oxidative stress status in plasma but not in plaque. Arterioscler Thromb Vasc Biol 2004, 24:136-140.

87. Barbagallo M, Dominguez LJ, Tagliamonte MR, Resnick LM, Paolisso G: Effects of vitamin $\mathrm{E}$ and glutathione on glucose metabolism: role of magnesium. Hypertension 1999, 34:1002-1006.

88. Sutken $E$, Inal M, Ozdemir F: Effects of vitamin E and gemfibrozil on lipid profiles, lipid peroxidation and antioxidant status in the elderly and young hyperlipidemic subjects. Saudi Med J 2006, 27:453-459.

89. Desideri G, Croce G, Tucci M, Passacquale G, Broccoletti S, Valeri L, Santucci A, Ferri C: Effects of bezafibrate and simvastatin on endothelial activation and lipid peroxidation in hypercholesterolemia: evidence of different vascular protection by different lipid-lowering treatments. J Clin Endocrinol Metab 2003, 88:5341-5347.

90. Manuel YK, Vinckx M, Vertommen J, Van GL, De Leeuw I: Impact of Vitamin E supplementation on lipoprotein peroxidation and composition in Type 1 diabetic patients treated with Atorvastatin. Atherosclerosis 2004, 175:369-376.

91. Duffy SJ, O'Brien RC, New G, Harper RW, Meredith IT: Effect of anti-oxidant treatment and cholesterol lowering on resting arterial tone, metabolic vasodilation and endothelial function in the human forearm: a randomized, placebo-controlled study. Clin Exp Pharmacol Physiol 2001, 28:409-418.

92. McDowell IF, Brennan GM, McEneny J, Young IS, Nicholls DP, McVeigh GE, Bruce I, Trimble ER, Johnston GD: The effect of probucol and vitamin $E$ treatment on the oxidation of low-density lipoprotein and forearm vascular responses in humans. Eur J Clin Invest 1994, 24:759-765.

93. De Caterina R, Cipollone F, Filardo FP, Zimarino M, Bernini W, Lazzerini G, Bucciarelli T, Falco A, Marchesani P, Muraro R, Mezzetti A, Ciabattoni G: Low-density lipoprotein level reduction by the 3-hydroxy-3-methylglutaryl coenzyme-A inhibitor simvastatin is accompanied by a related reduction of F2-isoprostane formation in hypercholesterolemic subjects: no further effect of vitamin E. Circ Cardiovasc Qual Outcomes 2002, 106:2543-2549.

94. Paolisso G, Gambardella A, Giugliano D, Galzerano D, Amato L, Volpe C, Balbi V, Varricchio M, D'Onofrio F: Chronic intake of pharmacological doses of vitamin $\mathrm{E}$ might be useful in the therapy of elderly patients with coronary heart disease. Am J Clin Nutr 1995, 61:848-852.

95. D'Arcangues C, Piaggio G, Brache V, Aissa RB, Hazelden C, Massai R, Pinol A, Subakir SB, Su-juan G, Study Group on Progestogen-induced Vaginal Bleeding Disturbances: Effectiveness and acceptability of vitamin E and low-dose aspirin, alone or in combination, on Norplant-induced prolonged bleeding. Contraception 2004, 70:451-462.

96. Gosai P, Liu J, Doyle RT, Johnson J, Carter R, Sica D, McKenney JM: Effect of omega-3-acid ethyl esters on the steady-state plasma pharmacokinetics of rosuvastatin in healthy adults. Expert Opin Pharmacother 2008, 9:2947-2953.

97. Abdul MI, Jiang X, Williams KM, Day RO, Roufogalis BD, Liauw WS, Xu H, Matthias A, Lehmann RP, McLachlan AJ: Pharmacokinetic and pharmacodynamic interactions of echinacea and policosanol with warfarin in healthy subjects. Br J Clin Pharmacol 2010, 69:508-515.

98. Mauro VF, Mauro LS, Kleshinski JF, Khuder SA, Wang Y, Erhardt PW: Impact of Ginkgo biloba on the pharmacokinetics of digoxin. Am J Ther 2003, 10:247-251.

99. Watson PD, Joy PS, Nkonde C, Hessen SE, Karalis DG: Comparison of bleeding complications with omega-3 fatty acids + aspirin + clopidogrelversus-aspirin + clopidogrel in patients with cardiovascular disease. Am J Cardiol 2009, 104:1052-1054.

100. Seely D, Kanji S, Yazdi F, Tetzlaff J, Singh K, Tsertsvadze A, Sears M, Tricco A, Ooi TC, Turek M, Tsouros S, Skidmore B, Daniel R, Ansari M: Dietary supplements in adults taking cardiovascular drugs. Rockville, MD: Agency for Healthcare Research and Quality; 2012. Publication No.12-EHC021-EF.

101. Foundation for Statistical Computing: $R$ : a language and environment for statistical computing. Vienna, Austria:; 2011.

102. Hajda J, Rentsch KM, Gubler C, Steinert H, Stieger B, Fattinger K: Garlic extract induces intestinal P-glycoprotein, but exhibits no effect on 
intestinal and hepatic CYP3A4 in humans. Eur J Pharm Sci 2010, 41:729-735.

103. Neil HA, Ceglarek U, Thiery J, Paul S, Farmer A, Holman RR: Impact of atorvastatin and omega-3 ethyl esters 90 on plasma plant sterol concentrations and cholesterol synthesis in type 2 diabetes: a randomised placebo controlled factorial trial. Atherosclerosis 2010, 213:512-517.

104. FDA should take further actions to improve oversight and consumer understanding. http://www.gao.gov/products/GAO-09-250.

105. Mills E, Wu P, Johnston BC, Gallicano K, Clarke M, Guyatt G: Natural health product-drug interactions: A systematic review of clinical trials. Ther Drug Monit 2005, 27:549-557.

106. Desai D, Hasan A, Wesley R, Sunderland E, Pucino F, Csako G: Effects of dietary supplements on aspirin and other antiplatelet agents: an evidence-based approach. Thromb Res 2005, 117:87-101.

107. Izzo AA, Di Carlo G, Borrelli F, Ernst E: Cardiovascular pharmacotherapy and herbal medicines: the risk of drug interaction. Int I Cardiol 2005, 98:1-14.

108. Skalli S, Zaid A, Soulaymani R: Drug interactions with herbal medicines. Ther Drug Monit 2007, 29:679-686.

109. Kennedy DA, Seely D: Clinically based evidence of drug-herb interactions: a systematic review. Expert Opin Drug Saf 2010, 9:79-124.

110. Egger M, Zellweger-Zahner T, Schneider M, Junker C, Lengeler C, Antes G: Language bias in randomised controlled trials published in English and German. Lancet 1997, 350:326-329.

doi:10.1186/2046-4053-1-26

Cite this article as: Kanji et al:: Interactions of commonly used dietary

supplements with cardiovascular drugs: a systematic review. Systematic Reviews 2012 1:26.

\section{Submit your next manuscript to BioMed Central and take full advantage of:}

- Convenient online submission

- Thorough peer review

- No space constraints or color figure charges

- Immediate publication on acceptance

- Inclusion in PubMed, CAS, Scopus and Google Scholar

- Research which is freely available for redistribution 